\title{
Effects of Gamma Radiation on Tertiary Butylhydroquinone and its Dosimetric Features
}

\author{
Hasan Tuner ${ }^{\mathrm{a}}$ and Mustafa Korkmaz \\ a Department of Physics, Faculty of Science, Balikesir University, Çağış, 10145, Balikesir, Turkey \\ $\mathrm{b}$ Department of Physics Engineering, Faculty of Engineering, Hacettepe University, Beytepe, \\ Ankara, 06800, Turkey
}

Reprint requests to Dr. H. T.; Fax: +90 31229920 37; E-mail: htuner@ hacettepe.edu.tr

Z. Naturforsch. 63a, $221-229$ (2008); received September 12, 2007

\begin{abstract}
The effects of gamma radiation on tertiary butylhydroquinone (TBHQ) are reported using electron spin resonance (ESR) spectroscopy. While unirradiated (control) TBHQ exhibited no ESR signal, irradiated one presented an ESR spectrum with five characteristic resonance peaks extended over a magnetic field region of $1.5 \mathrm{mT}$. Variations of the heights of the resonance peaks and the spectrum area as function of the microwave power, applied dose, storage time, and temperature were studied. The kinetic features and spectroscopic parameters of the species responsible for the experimental ESR spectrum were investigated by annealing studies performed at four different temperatures and simulation calculations, respectively. A model based on the presence of two species having different kinetic and spectroscopic features was found to describe best the experimental results. The dosimetric potential of TBHQ was also investigated, and it was concluded that the discrimination of irradiated TBHQ from unirradiated one was possible even long after the radiation treatment, and that radiation doses above $5 \mathrm{kGy}$ could be measured with an accuracy better than $3 \%$ by using TBHQ. Two tentative radical species were proposed.
\end{abstract}

Key words: Radiation; TBHQ; Electron Spin Resonance (ESR); Radical.

\section{Introduction}

Tertiary butylhydroquinone (TBHQ), i. e. 2-(1,1-dimethylethyl)-1,4-benzenediol, is a type of phenol. It is a derivative of hydroquinone, substituted with a tertbutyl group. TBHQ has recently been regarded as the best general-purpose food grade antioxidant (E319) and has replaced the traditional antioxidants in oil or fats and oil-containing foods in many countries. It is used as varnish, lacquer, resin and especially oil filed additives, as a fixative in perfumery to reduce the evaporation and to improve the stability, and as stabilizer to inhibit auto-polymerization of organic peroxides.

Although the antioxidant potency of TBHQ has been widely studied in different foods [1 - 11], its stability against radiation has not yet been investigated, neither as a pure compound nor in TBHQ-containing irradiated foods. ESR spectroscopy is one of the powerful analytical methods used in the detection of irradiated foods standardized by the European Committee for Standardization (CEN) in EN-1787 and EN-13708. Therefore, the aims of the present work are: first to investigate the radiation sensitivity of solid TBHQ toward gamma rays in the irradiation dose range of $1-$<smiles>CC(C)(C)c1ccccc1O</smiles>

Fig. 1. Structure of TBHQ.

34 kGy through a detailed ESR study carried out at room and high temperatures, the kinetic features of the radical species produced after irradiation of TBHQ and, finally, to determine its dosimetric potential for gamma radiation dose measurement.

\section{Materials and Methods}

\subsection{Materials}

Tertiary butylhydroquinone was kindly supplied by the GMT Food Ingredients Company (Istanbul), and 


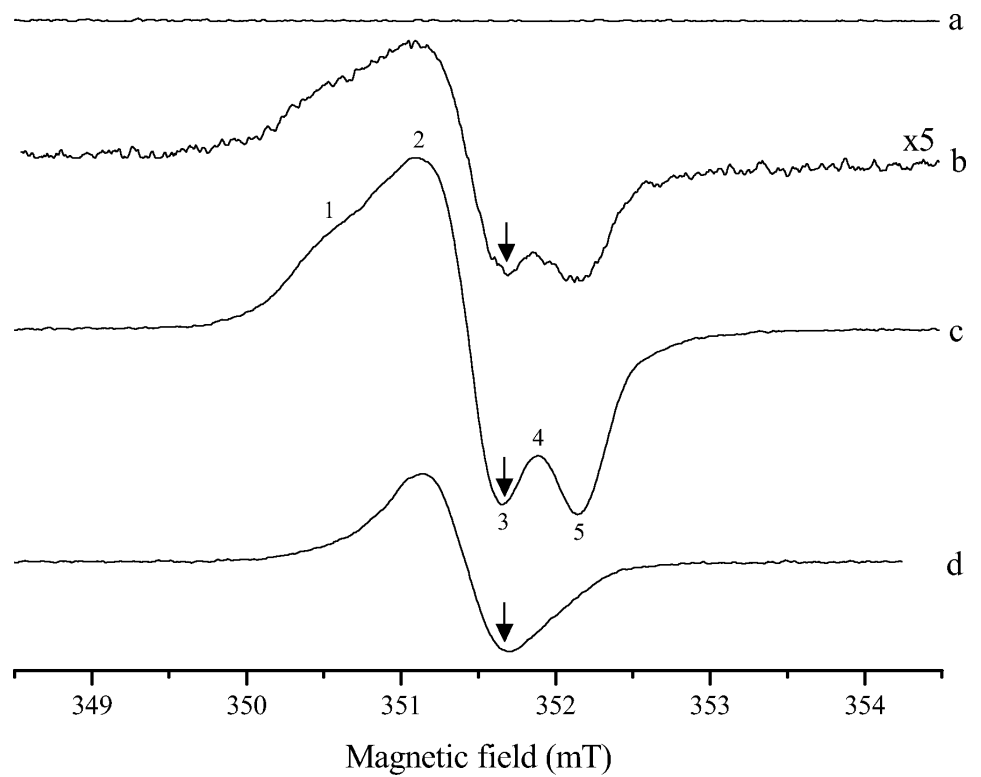

Fig. 2. Spectra of unirradiated and irradiated TBHQ samples with assigned peak numbers recorded at room temperature. (a) Unirradiated; (b) and (c) just after irradiation at $1 \mathrm{kGy}$ and $10 \mathrm{kGy}$, respectively; (d) 100 days after irradiation at $10 \mathrm{kGy}$. Arrows indicate the position of the DPPH resonance line $(g=2.0036)$. it was used without further treatment. The electron spin resonance (ESR) investigation was principally performed on TBHQ of food grade. Some experiments were also carried out on TBHQ of spectroscopic grade, but no pattern changes were observed in the ESR spectra recorded at low and high temperatures. TBHQ is a white to light tan crystalline powder with a melting point between 126.5 and $128.5^{\circ} \mathrm{C}$. Its molecular structure is presented in Figure 1.

\subsection{Irradiation}

All irradiations were performed at room temperature $(290 \mathrm{~K})$ using a ${ }^{60} \mathrm{Co}$ gamma cell 220 as ionizing radiation source (dose rate $1.41 \mathrm{kGy} / \mathrm{h}$ ) at the Sarayköy Nuclear Research Center of the Turkish Atomic Energy Agency in Ankara in the dose range of $1-34 \mathrm{kGy}$. Sealed glass vials containing about $3 \mathrm{~g}$ TBHQ were exposed to successively increasing doses of radiation at constant intensity. The source was calibrated against a Fricke ferrous sulfate dosimeter, and the dose rate in the irradiated samples was calculated by applying appropriate corrections on the basis of photon mass attenuation and energy absorption coefficient for the sample and dosimetric solution.

\subsection{Instrumentation}

ESR measurements were performed using a BRUKER EMX 131 X-band spectrometer equipped with a cylindrical cavity (ER 4119HS). Samples were positioned in the microwave cavity by a quartz ESR tube of $4 \mathrm{~mm}$ inside diameter. Sample temperatures inside the microwave cavity were monitored by a digital temperature control system (BRUKER BVT3000 ER4131-VT) with an accuracy of $\pm 0.5 \mathrm{~K}$ at the site of the sample. The evolution of the ESR signal with the applied microwave power, received radiation dose, storage time and temperature were followed by both calculating the areas of the ESR spectra and measuring the heights of characteristic resonance peaks of the recorded experimental spectra. A DPPH (1,1-diphenyl-2-picrylhydrazyl) sample was used as standard ( $g=$ 2.0036).

A set of four different samples irradiated at a dose of $10 \mathrm{kGy}$ was annealed at $315,320,323$ and $325 \mathrm{~K}$ for predetermined times, and the signal intensity data obtained were used to calculate the decay characteristics of radical species responsible for the ESR spectra of gamma-irradiated TBHQ.

\subsection{Data Analysis}

Evaluations were performed using data derived from five different measurements carried out on five different samples prepared from the same TBHQ bath irradiated at a given radiation dose. Analyses of experimental data were accomplished by adopting a model based on two radical species exhibiting different spectroscopic features. Numerical spectrum simulations were carried out using home-made software. Five different mathematical functions were tried to analyze the 


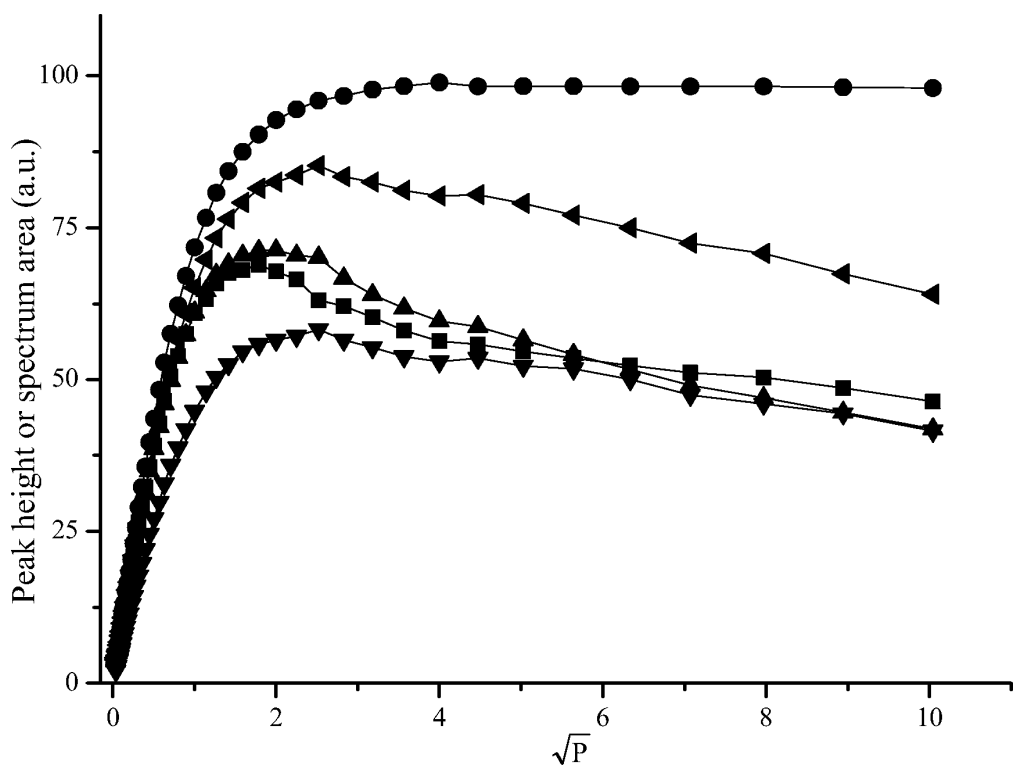

Fig. 3. Variations of the heights of assigned peaks and the spectrum area with microwave power at room temperature for a sample irradiated at a dose of $10 \mathrm{kGy}$. ( $\mathbf{\square})$ Peak 2; ( $\mathbf{(})$ peak 3;

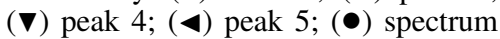
area. Lines are obtained simply by joining data points. applied dose-response data and thus to determine the dosimetric potential of TBHQ.

\section{Results and Discussion}

While unirradiated TBHQ samples exhibited no ESR signal, irradiated samples presented characteristic ESR spectra consisting of four resonance peaks well developed at the high dose with a shoulder at low magnetic fields (Figs. 2a, b, c). As is seen in Fig. 2b, the resonance peaks are not well developed at the low radiation dose. Variations of the spectrum pattern, height of the assigned resonance peaks and area under the spectra calculated by double integration were observed as a function of the microwave power, applied radiation dose, storage time and temperature.

\subsection{Variations of the Peak Heights and the Spectrum Area with Microwave Power}

At room temperature, the peak heights and spectrum area varied with the microwave power as presented in Figure 3. It is seen that heights of the assigned peaks and the spectrum area increased rather linearly at low power $(0.001-1.0 \mathrm{~mW})$ except for peak 4 , which deviated from linear variation at still lower powers. Nevertheless, peak 4 was expected to be heavily influenced by the presence of the peaks 3 and 5. All assigned peaks exhibited the characteristic behaviour of homogeneously broadened resonance lines above $4.0 \mathrm{~mW}$, but their variations are different. Although, the heights of the peaks 2 and 3 experience relatively sharp decreased above about $4 \mathrm{~mW}$, peak 5 varied smoothly above this power. The differences in the increase and decrease rates of microwave saturation curves at low and high microwave power (Fig. 3) were considered as implying the presence of more than one radical species in gamma-irradiated TBHQ.

The microwave saturation behaviour of assigned peaks was also studied at low temperature $(130 \mathrm{~K})$, and the results relevant to the heights of the peaks 2 and 3 and the spectrum area are presented in Figure 4. As is expected the assigned resonance peaks started to saturate at still low power, that is above $0.25 \mathrm{~mW}$ microwave power, and the peaks 2 and 3 experienced similar linear increases at low temperature as they do at room temperature. At $130 \mathrm{~K}$, the homogeneously broadened features of the studied resonance peaks disappeared above $0.25 \mathrm{~mW}$; instead, the resonance peaks exhibited the features of inhomogeneously broadened resonance lines. Experimental microwave saturation data obtained at room temperature $(290 \mathrm{~K})$ and at $130 \mathrm{~K}$ for assigned peaks were fitted to linear functions in the microwave power ranges of $0.001-$ $1.0 \mathrm{~mW}$ and $0.05-0.25 \mathrm{~mW}$, respectively, to compare numerically the slopes of the linear increases in the resonance heights and the spectrum area. It was found that at low microwave powers the height of the assigned peaks do not increase with the same slopes, and that the slopes calculated from $130 \mathrm{~K}$ data are larger than those obtained at room temperature for the same peaks. 


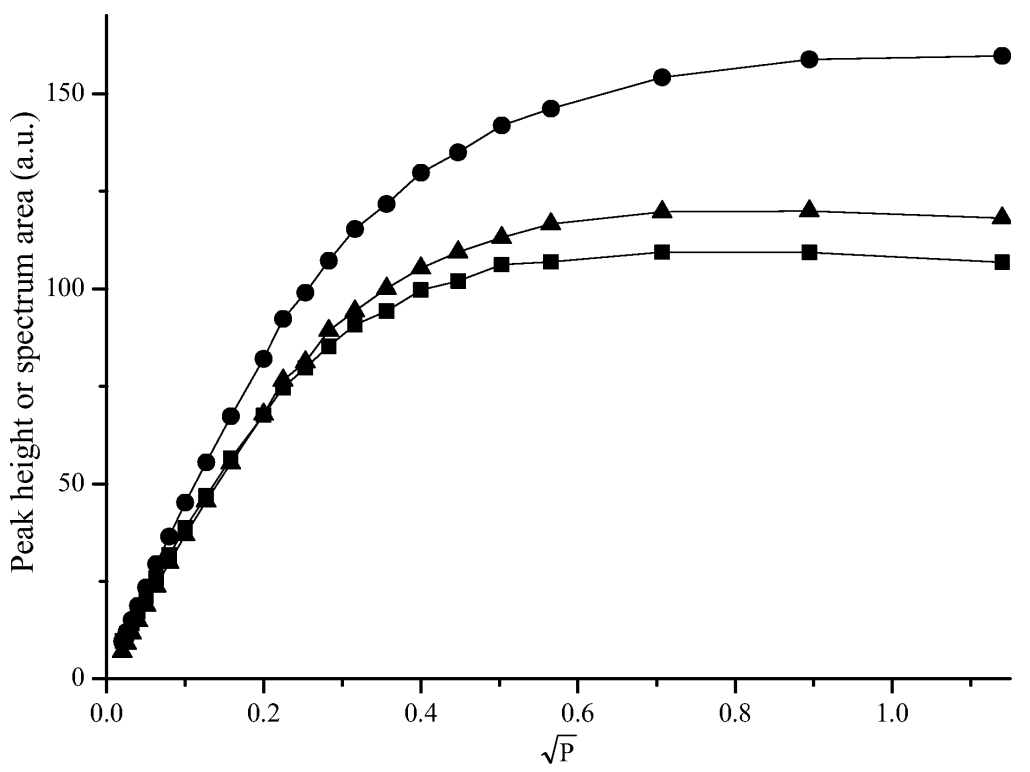

Fig. 4. Variations of the heights of assigned peaks and the spectrum area with microwave power at $130 \mathrm{~K}$ for a sample irradiated at a dose of $10 \mathrm{kGy}$.

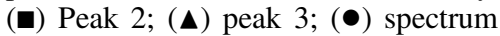
area. Lines are obtained simply by joining data points.

\subsection{Variations of the Peak Heights and the Spectrum Area with Temperature}

A variable temperature study was also performed using a sample irradiated at $10 \mathrm{kGy}$ in the temperature range of $130-330 \mathrm{~K}$. The sample was first cooled to $130 \mathrm{~K}$, starting from $290 \mathrm{~K}$ with an increment of $20 \mathrm{~K}$. Then the temperature was increased up to $330 \mathrm{~K}$ with the same increment. The spectra were recorded $5 \mathrm{~min}$ after setting the temperature. No pattern changes in the ESR spectra were observed below room temperature except expected reversible Curie changes in the height of the studied assigned peaks. However, sharp irreversible decreases in the heights of all peaks related with an important radical decay were observed above $320 \mathrm{~K}$. The results obtained for peak 2 and the spectrum area are presented in Fig. 5 as an example for these variations. Observed drastic decreases in the peak heights and the spectrum area motivated us to perform annealing studies above room temperature to determine kinetic features of the radical species responsible for the experimental ESR spectrum of gamma-irradiated TBHQ.

\subsection{Long-Term Decays in the Peak Heights at Room Temperature}

A sample irradiated by a dose of $15 \mathrm{kGy}$ was stored in air at room temperature. The evolution of the ESR spectrum was observed over a period of 100 days by recording the spectra in regular time intervals without changing the position of the sample in the microwave cavity. Similar studies were performed on samples irradiated at high doses, and similar variations in the peak heights were obtained. The results relevant to peaks 2, 3,5 and the spectrum area are given in Figure 6. All peaks and the spectrum area experience fast decreases at the beginning of the storage period, followed by a slow decay. In accordance with the results derived from microwave saturation studies, spectrum simulation calculations and kinetic studies at high temperatures, the experimental spectrum area decay data derived by double integration were fitted to functions consisting of the sum of two exponentially varying functions of storage time as given by

$$
I(t)=I_{\mathrm{AO}} \mathrm{e}^{-k_{\mathrm{A}} t}+I_{\mathrm{BO}} \mathrm{e}^{-k_{\mathrm{B}} t},
$$

assuming that the radicals responsible for the experimental spectrum follow by first-order decay kinetics. In (1) $k_{\mathrm{A}}$ and $k_{\mathrm{B}}$ represent the decay constants, $I_{\mathrm{AO}}$ and $I_{\mathrm{BO}}$ are two constants proportional to the initial concentrations of the involved radical species, $I(t)$ being the spectrum area calculated from the recorded experimental spectrum by double integration. $k_{\mathrm{A}}, k_{\mathrm{B}}, I_{\mathrm{AO}}$ and $I_{\mathrm{BO}}$, best describing the experimental spectrum area data, were obtained by curve fitting. The results are presented in Table 1. Similar curve fitting procedures were performed using experimental peak height values derived for assigned peaks, and similar results 

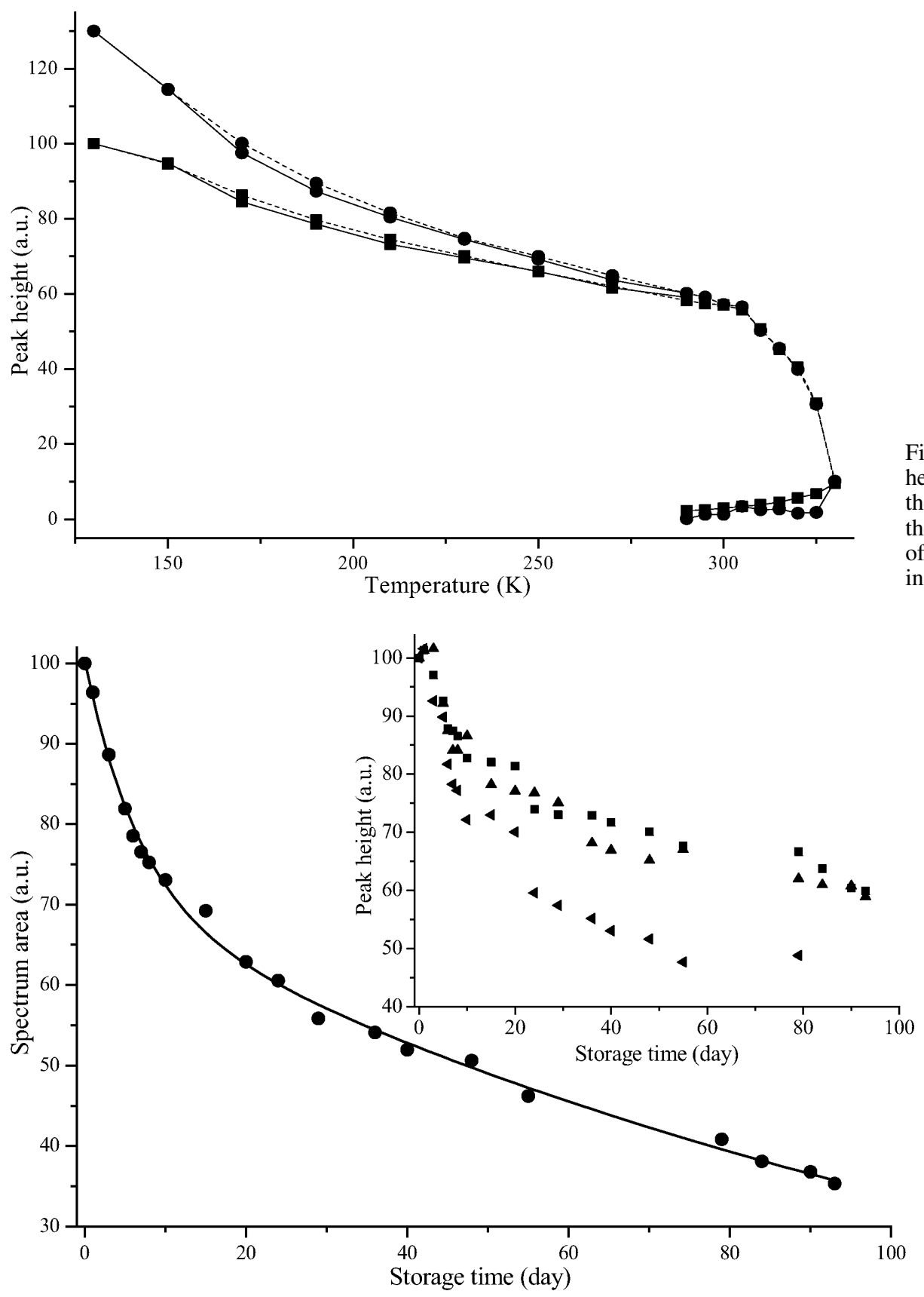

Fig. 5. Variations of the heights of peak 2 (ם) and the spectrum area $(\bullet)$ with the temperature in the range of $130-325 \mathrm{~K}$. (-) Cooling; (---) heating.
Fig. 6. Variations of the heights of peaks $[(\boldsymbol{\square})$ peak $2 ;(\boldsymbol{\Delta})$ peak $3 ;(\triangleleft)$ peak 5 (inset)] and the spectrum area $(\bullet)$ with storage time. Solid line is calculated from parameters given in $\mathrm{Ta}-$ ble 1. were obtained for $k_{\mathrm{A}}, k_{\mathrm{B}}, I_{\mathrm{AO}}$ and $I_{\mathrm{BO}}$. Theoretical decay data, calculated using parameter values given in Table 1 for the spectrum area, are presented in Fig. 6 for comparison. By inspection of Fig. 6 it is seen that the model based on two radical species of different decay characteristics describes fairly well the experimental room temperature decay data obtained for gamma- irradiated TBHQ, and that species A is responsible for the fast decrease observed at the beginning of the storage period.

\subsection{Dosimetric Features of $T B H Q$}

In the radiation dose measurements, the sensitivity of the ESR technique is considered in terms of the limit 


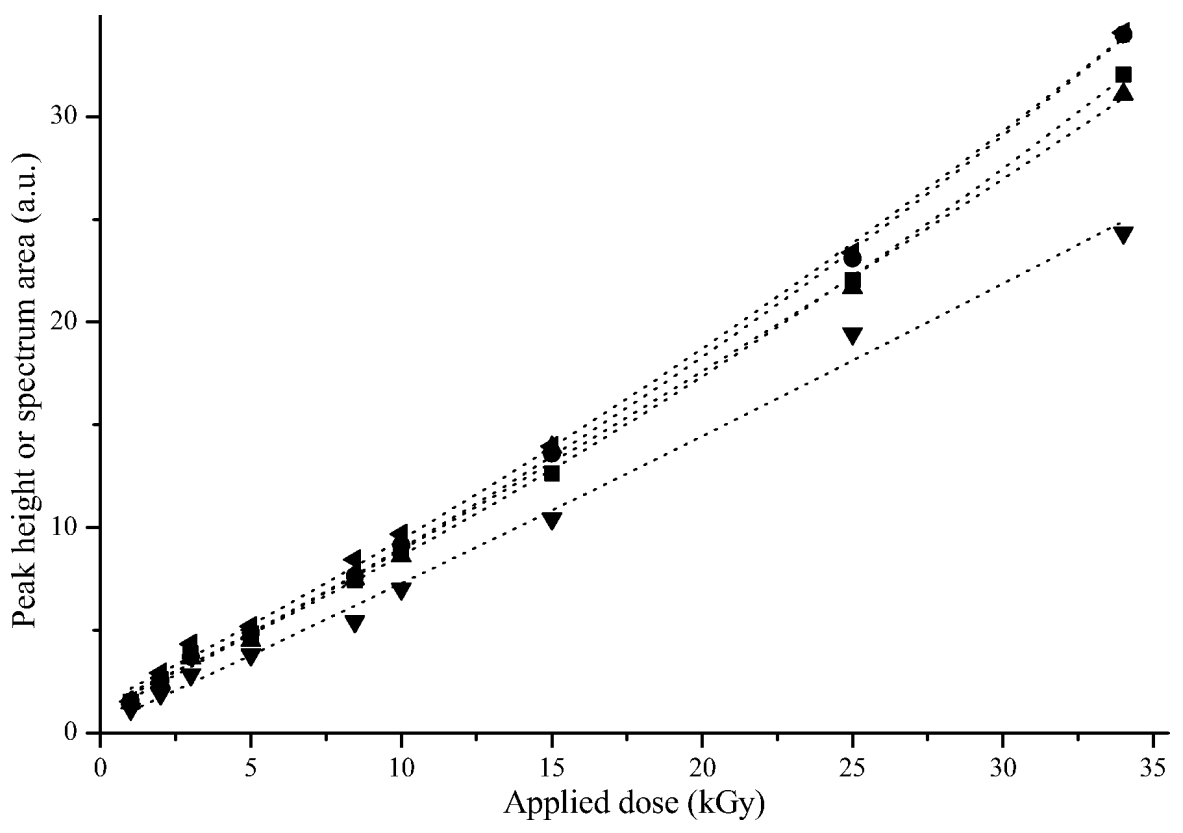

Fig. 7. Variations of the peak heights and spectrum area with applied dose. (ロ) Peak 2;

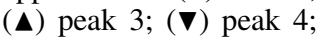
(४) peak 5; (•) 1. spectrum area. Dotted lines: theoretical results obtained by fitting experimental data to a function comprising a linear and a quadratic dose terms.
Table 1. Room temperature decay constants and initial percent contents calculated from long-term spectrum area decay data for contributing radical species.

\begin{tabular}{cccc}
\hline $\begin{array}{c}\text { Radical } \\
\text { species }\end{array}$ & $\begin{array}{c}\text { Initial percent } \\
\text { content }\end{array}$ & $\begin{array}{c}\text { Decay constant } \\
\left(\mathrm{d}^{-1}\right) \cdot 10^{4}\end{array}$ & $\begin{array}{c}\text { Correlation } \\
\text { coefficient }\end{array}$ \\
\hline A & $0.71 \pm 0.05$ & $73.5 \pm 1.5$ & \\
B & $0.29 \pm 0.04$ & $1489.8 \pm 12.1$ & 0.9974 \\
\hline
\end{tabular}

of detection (LOD) and limit of quantification (LOQ), which are predicted by the $S / N=3$ and $S / N=10$ criteria, respectively. They have been determined to be 0.1 and $1.0 \mathrm{kGy}$ in the case of gamma-irradiated TBHQ. Since $25 \mathrm{kGy}$ is the established and accepted applied dose limit in the radiosterilization of pharmaceuticals, it was concluded that the discrimination of irradiated TBHQ from unirradiated one is possible even long after the radiation treatment.

Beside qualitative detection, ESR spectroscopy can also be used for dose estimation. However, the choice of appropriate mathematical functions used to describe the dose-response data is important. Five different functions (linear, linear + quadratic, power, exponential and sum of two exponentials) proposed previously in different works for observed dose estimation in processed food $[12,13]$ and irradiated pharmaceuticals $[14,15]$ have been tried in the present work to describe experimental dose-response data (Fig. 7), which are the mean of single determinations on three different samples. It should be noted that no attempt has been
Table 2. Parameter values $a, b$ and $c$ of the function $I=$ $a+b D+c D^{2}$ and correlation coefficient (numbers in parenthesis) best describing experimental dose-response data.

\begin{tabular}{rrrrrr}
\hline \multicolumn{5}{c}{ Resonance peaks } \\
& \multicolumn{1}{c}{2} & \multicolumn{1}{c}{3} & \multicolumn{1}{c}{4} & \multicolumn{1}{c}{5} & \multicolumn{1}{c}{$d I$} \\
\hline$a$ & 1.3199 & 0.8767 & 0.3883 & 1.4152 & 1.1506 \\
& \pm 0.0020 & \pm 0.0012 & \pm 0.0015 & \pm 0.0025 & \pm 0.0011 \\
$b$ & 0.6603 & 0.7687 & 0.7033 & 0.7367 & 0.7092 \\
& \pm 0.0012 & \pm 0.0013 & \pm 0.0018 & \pm 0.0020 & \pm 0.0014 \\
& 0.0071 & 0.0034 & 0.0005 & 0.0064 & 0.0074 \\
& \pm 0.0009 & \pm 0.0010 & \pm 0.0001 & \pm 0.0012 & \pm 0.0015 \\
& $(0.9992)$ & $(0.9984)$ & $(0.9954)$ & $(0.9988)$ & $(0.9995)$ \\
\hline
\end{tabular}

$\overline{d I}$ spectrum area calculated by double integration.

made to force the curves to pass through zero. Interestingly, the function consisting of the sum of a linear and a quadratic applied dose term, $I=a+b D+c D^{2}$, was found to correlate well with the experimental data obtained for assigned peaks, although the correlation was much better for the spectrum area calculated by double integration than the correlation with the heights of the investigated resonance peaks in the dose range of $1-$ $34 \mathrm{kGy}$. Parameter values and correlation coefficients calculated through fitting experimental dose-response data to the function $I=a+b D+c D^{2}$ are presented in Table 2. Similar calculations were also performed using the other functions mentioned above, but the results relevant with these functions are not given here.

The utility of the mathematical function describing best the experimental dose-response data, namely 


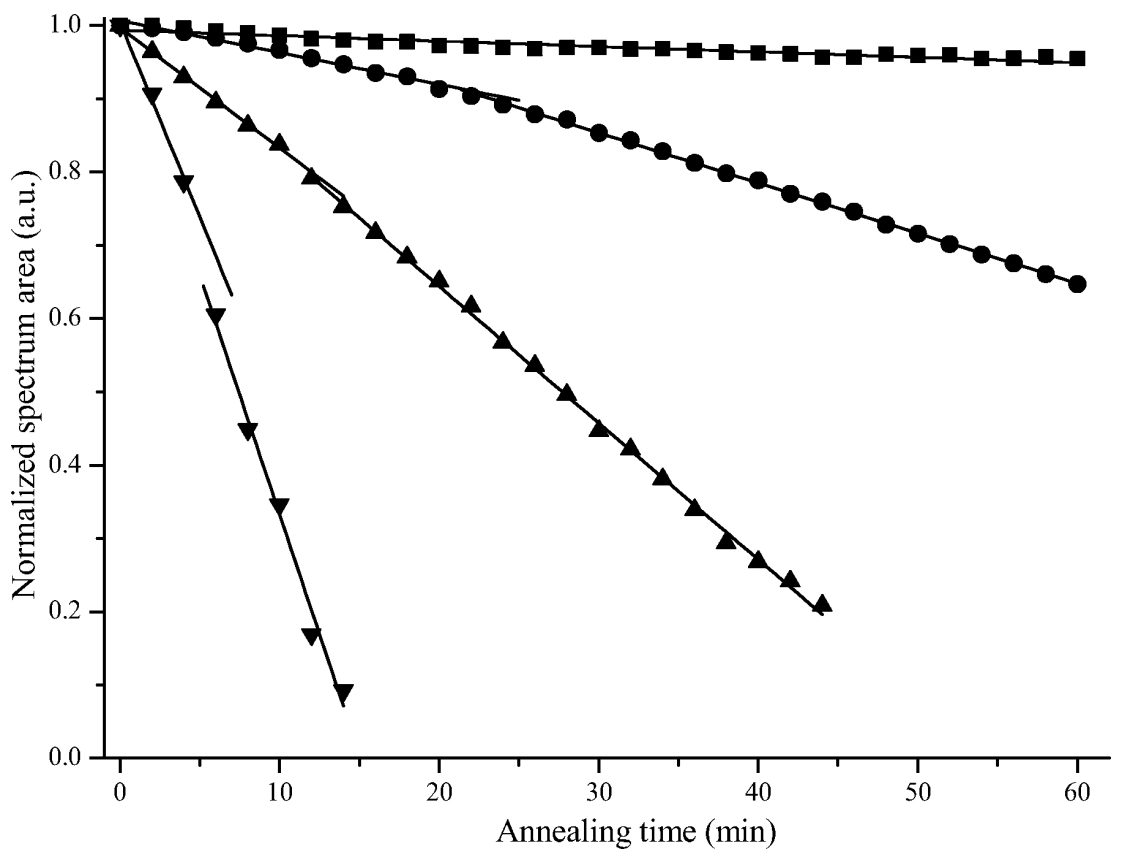

Fig. 8. Variations of the spectrum area with annealing time at four different temperatures. (匹) $315 \mathrm{~K}$; (•) $320 \mathrm{~K}$; (४) $323 \mathrm{~K} ;(\mathbf{\nabla}) 325 \mathrm{~K}$. a function comprising a linear and quadratic term of applied doses, was tested by calculating the interpolated doses. To achieve this goal, the measured peak heights or calculated spectrum area were entered into mathematical functions, and corresponding doses $\left(D_{\mathrm{c}}\right)$ were calculated for each resonance peak and spectrum area. The results are presented as the ratios of calculated $\left(D_{\mathrm{c}}\right)$ to measured dose $\left(D_{\mathrm{m}}\right)$ versus the measured dose $\left(D_{\mathrm{m}}\right)$. From inspection of the results obtained it was concluded that the dose $5 \mathrm{kGy}$ or higher can be estimated with an accuracy better than $3 \%$, if the spectrum area and/or peak height values of peak 2 are used. However the accuracy at lower doses was not good. Therefore, TBHQ can be a suitable material to estimate the applied radiation dose just after the irradiation, if its dose-response data are calibrated properly, using a function comprising a linear and a quadratic dose term.

\subsection{Effects of Annealing on Signal Intensities}

Investigation of the variations of ESR signal intensities in annealed samples would be interesting from a kinetic point of view of the contributing radical species. Irreversible decreases of the intensities at high temperatures would be expected to originate from the decay of the species. Beside, the decay rate of these species should depend on the sample temperature. To test this idea and to get more insight into the decay processes of the studied resonance peaks, some irradiated TBHQ samples were annealed at the temperatures $315,320,323$ and $325 \mathrm{~K}$ below their melting temperature for predetermined times up to $60 \mathrm{~min}$. Signal intensities measured from the recorded spectra of the samples placed in heated nitrogen gas flowing through the microwave cavity were used for this purpose. The intensities were normalized to the intensity calculated from the first spectrum recorded 5 min after positioning the sample in the microwave cavity for establishing thermal equilibrium. Variations of the spectrum area at four annealing temperatures are given in Fig. 8 for a sample irradiated at a dose of $10 \mathrm{kGy}$. The heights associated with the peaks 2, 3, 4 and 5 were found to exhibit similar decay characteristics. As is expected, the higher the temperature the faster was the decay of the spectrum area and peak heights. Radical species were found to decay differently at room and at annealing temperatures (Figs. 6 and 8). Although at room temperature species responsible for the experimental ESR spectrum followed an exponential decay with a very small decay constant (Table 1), they were found to exhibit a faster decay at $320 \mathrm{~K}$ and above this temperature. Moreover, the decay at high temperatures presents a biphasic character likely due to the change in the cage effect of the TBHQ solid matrix above a certain temperature (about $320 \mathrm{~K}$, Fig. 8). As is seen from the DSC thermogram given in Fig. 9 the increase in the 


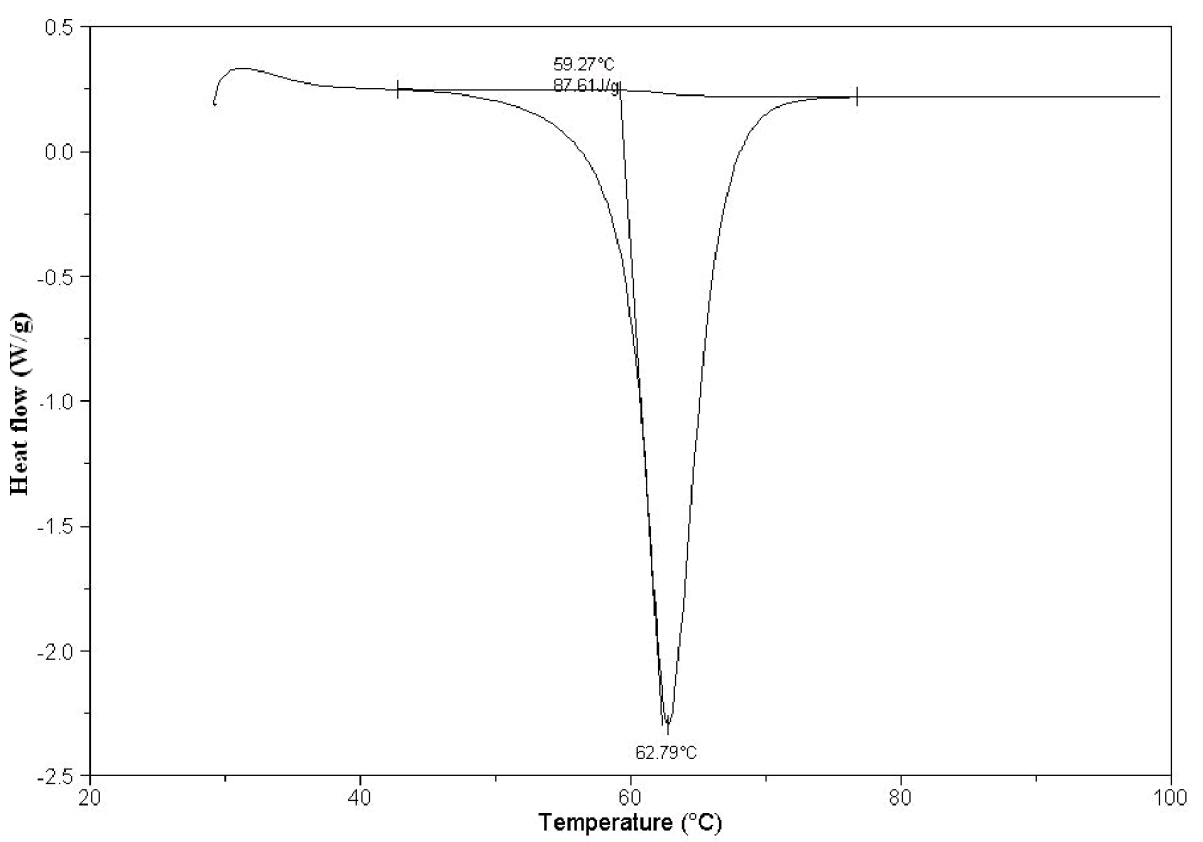

Fig. 9. DSC thermogram of TBHQ. heat flow transferred to the sample indicate a softening of the TBHQ solid matrix, which causes a strong increased in the radical-radical recombination and/or disproportionation reaction rates above $320 \mathrm{~K}$. Efforts made to describe the spectrum area and peak height decay data using first- and/or second-order decay expressions related with more than one radical species of different concentrations failed due to the annealing time-dependent softening of the TBHQ solid matrix.

\subsection{Simulation Calculations}

The ESR parameter of the species responsible for the experimental spectrum of gamma-irradiated TBHQ was also determined by simulation calculations based on a model assuming the presence of two radical species with different spectroscopic features. Signal intensity data derived from room temperature ESR spectra of a sample irradiated at a dose of $10 \mathrm{kGy}$ were used as input to run the home-made simulation program. The results of the calculations are presented in Table 3 . The theoretical spectrum of each species and their sum were also calculated. They are given in Fig. 10 together with their experimental counterpart for comparison. As is seen, the agreement between the experimental and theoretical sum spectrum is relatively good. Species A with its relatively high content and big linewidth dominates the observed ESR spectrum of gamma-irradiated
Table 3. Spectroscopic parameters calculated for contributing radical species $\mathrm{A}$ and $\mathrm{B}$.<smiles>CC(C)(C)c1ccccc1O</smiles>

B<smiles>CC(C)(C)c1c(O)cccc1O</smiles>

Percent content: 0.567 Linewidth (mT): 0.715 $g$ Value: $g_{\text {iso }}=2.0048$

Percent content: 0.433 Hyperfine splitting (mT): $A_{x x}=0.492$ $A_{y y}=0.690$ $A_{z z}=0.723$

$g$ Values:

$g_{x x}=2.0047$

$g_{y y}=2.0035$

$g_{z z}=2.0059$ Linewidth (mT): 0.359

TBHQ. Species A has minor contributions to peaks 1 and 5. This means that these two peaks can be used to monitor the behaviour of species $\mathrm{B}$.

Species A and B are believed to be created by homolytic dissociations of $\mathrm{O}-\mathrm{H}$ bonds and one of the $\mathrm{C}-\mathrm{H}$ bonds at position 5 or 6 of the benzene ring, respec- 


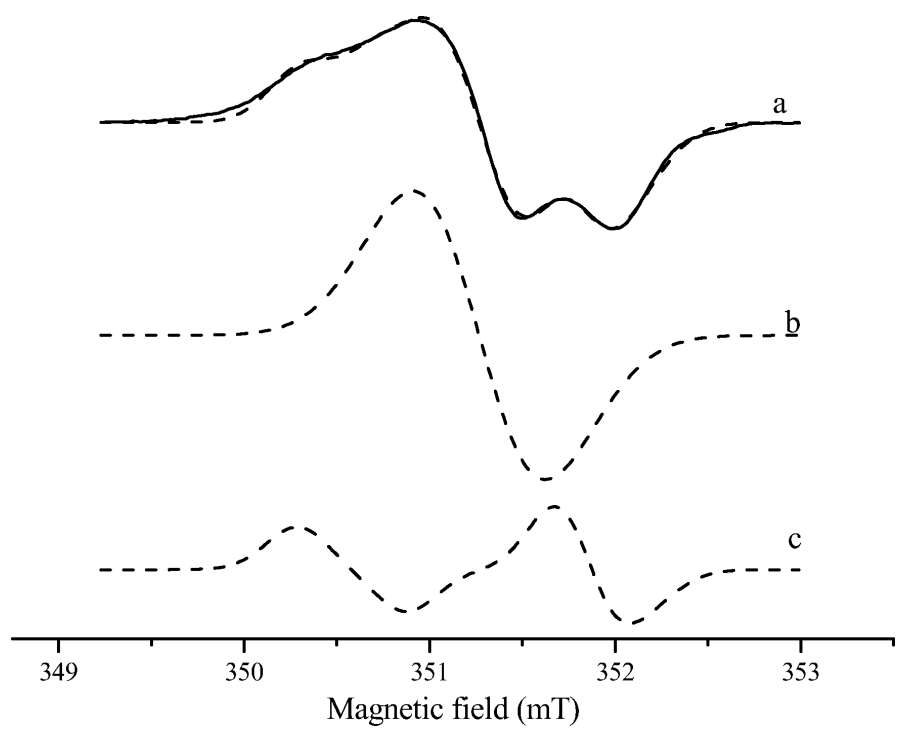

Fig. 10. Experimental (solid line) and theoretical (dashed lines) ESR spectra calculated using parameter values given in Table 3. (a) Sum spectra; (b) species A; (c) species B. tively. Dissociation of O-H bonds creates species with unpaired electrons localized at oxygen atoms, and dissociation of $\mathrm{C}-\mathrm{H}$ bonds creates species with unpaired electrons localized at carbon atoms. While neighbouring protons to oxygen atoms give rise to line broadening in species A, a proton attached to the nearest neighbour carbon atom of the benzene ring produces an orientation-dependent hyperfine splitting in species B, whose unpaired electron is localized on a carbon atom.

\section{Conclusion}

- Gamma radiation produces damage in TBHQ, and the extent of the damage increases with the increase of the radiation dose.

- Two radical species of different kinetic features

[1] E. R. Sherwin and J. W. Thompson, Food Technol. 21, 912 (1967).

[2] M. H. Chahine and R.F. Macneill, J. Am. Oil Chem. Soc. 51, 37 (1974).

[3] B. J.F. Hudson, Food Antioxidants, Elsevier Science Publisher Ltd., New York 1990, p. 1-18, 64.

[4] R. O. Dunn, Fuel Process. Technol. 82, 381 (2005).

[5] E. A. Decer, K. A. Warner, and M. Richards, J. Agric. Food Chem. 53, 4303 (2005).

[6] F. Shahidi and P. K. Wanasundara, Crit. Rev. Food Sci. 31, 67 (1992).

[7] A. Valenzuela, J. Sanhueza, and S. Nieto, Biol. Res. 36, 291 (2003).

[8] R. Anton, S. Barlow, D. Boskou, L. Castle, R. Crebelli, W. Dekant, K. H. Engel, S. Forsythe, W. Grunow, J. C. and different spectroscopic parameters are responsible for the observed ESR spectra.

- At room temperature, decays of radical species are slow. Therefore, irradiated TBHQ can be distinguished from unirradiated one even after 90 days of storage.

- The decay of peak heights or spectrum area at high annealing temperature exhibits a biphasic character attributable to the change produced in the cage effect of the solid matrix above $320 \mathrm{~K}$.

- TBHQ can be used as a dosimetric material due to its relatively high radiation sensitivity. However, this feature of TBHQ produces a severe drawback in irradiation of TBHQ-containing foods and in its radiosterilization.

Larsen, C. Leclercq, W. Mennes, M. R. Milana, I. Rietjens, K. Svensson, P. Tobback, and F. Toldrá, Europ. Food Safety Authority (EFSA) J. 84, 1 (2004).

[9] Y. B. C. Man and L. Jailong, J. Food Lipids 6, 117 (1999).

[10] J. D. Dziezak, Food Technol. 40, 94 (1986).

[11] T. Asap and A. Augustine, J. Am. Oil Chem. Soc. 37, 1045 (1986).

[12] J. P. Basly, I. Longy, and M. Bernard, Anal. Chim. Acta 359, 107 (1998).

[13] J. P. Basly, I. Basly, and M. Bernard, J. Pharm. Biomed. Anal. 17, 871 (1998).

[14] M. F. Desrosiers, G. L. Wilson, C. R. Hunter, and D. R. Hutton, Appl. Radiat. Isot. 42, 613 (1991).

[15] M. F. Desrosiers, Appl. Radiat. Isot. 42, 617 (1991). 